\title{
Theorien zur Mediennutzung bei der Gruppenarbeit
}

\author{
Schwabe, Gerhard
}

Posted at the Zurich Open Repository and Archive, University of Zurich ZORA URL: https://doi.org/10.5167/uzh-57149

Book Section

Accepted Version

Originally published at:

Schwabe, Gerhard (2001). Theorien zur Mediennutzung bei der Gruppenarbeit. In: Schwabe, Gerhard; Streitz, N; Unland, Rainer. CSCW Kompendium - Lehr- und Handbuch zur computerunterstützten Gruppenarbeit. Berlin / Heidelberg, Deutschland: Springer, 0. 


\section{Theorien zur Mediennutzung bei der Gruppenarbeit}

\section{Einleitung}

Dieses Kapitel stellt Theorien zur Mediennutzung bei der Gruppenarbeit vor. Während Theorien wie die Media-Richness-Theory in der deutschen betriebswirtschaftlichen Forschung sich inzwischen etabliert haben (vgl. z.B. [Reichwald et al 1998]), sind die TIP-Theorie, die Adaptive Structuration Theory oder die Media Synchronicity Theorie in Deutschland noch weitgehend unbekannt, obwohl sie spannende neue Einsichten zu dem Zusammenspiel von elektronischen Medien und Gruppen in einem organisatorischen Kontext geben.

\section{Die Media-Richness-Theorie}

Die Media-Richness-Theorie [Daft und Lengel 1984, 1986] verbindet die Medienwahl mit der Aufgabe, die die beteiligten Akteure gemeinsam lösen wollen. Sie teilt Aufgaben danach ein, wie unsicher ${ }^{1}$ sie sind und wie mehrdeutig. Unsichere Aufgaben könnte man optimal lösen, wenn alle benötigten Informationen vorhanden wären.

Mehrdeutige Aufgaben lassen sich auch durch sehr viel Information nicht lösen. Vielmehr unterliegen sie der Interpretationsfähigkeit der Akteure, die zu einem gemeinsamen Verständnis eines Sachverhalts kommen müssen. Beispielsweise ist das Verfassen einer Unternehmensstrategie eine mehrdeutige Aufgabe, weil sich die Akteure dabei auf ein gemeinsames Verständnis der Rolle des Unternehmens im Markt verständigen und dafür die relevanten Einflußfaktoren erst definieren müssen. Bei mehrdeutigen Aufgaben sucht man Variablen; bei

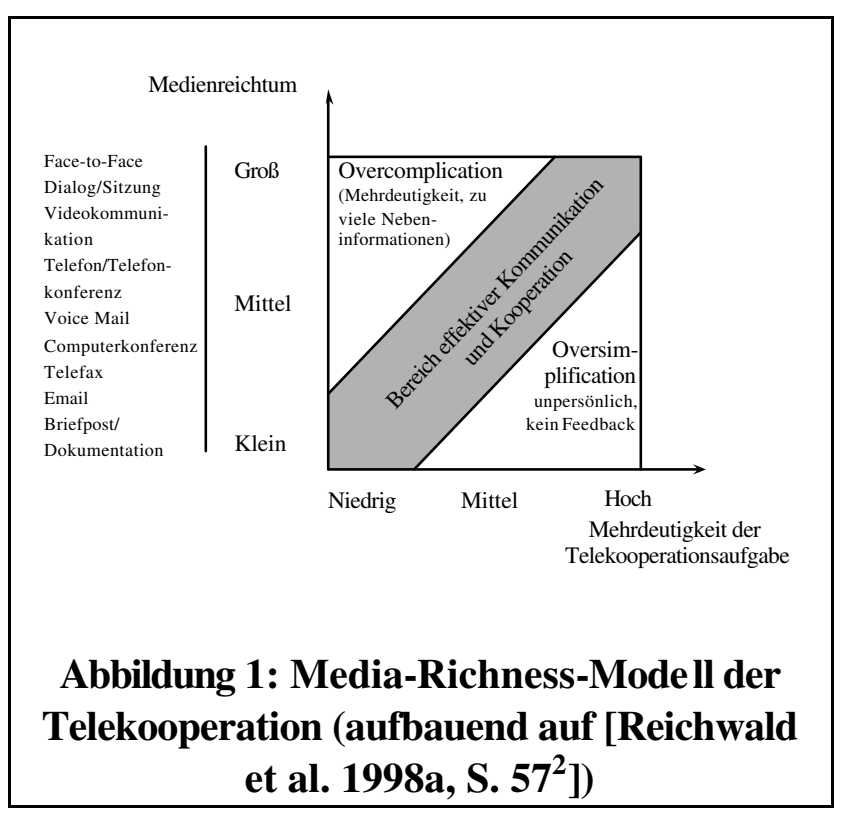
unsicheren Aufgaben Variablenwerte.

Die ursprüngliche Fassung der MediaRichness-Theorie von Daft und Lengel [1984, 1986] empfiehlt, für unsichere Aufgaben Medien $\mathrm{zu}$ verwenden, die viel Information vermitteln (z.B. schriftliche Berichte), und für mehrdeutige Aufgaben Medien $\mathrm{zu}$ verwenden, die 'reich' sind (z.B. Sitzungen). Den Reichtum ('Richness') eines Mediums kann man daran messen, wie unmittelbar das Feedback ist, wieviele Kanäle wieviele Hinweise geben, wie persönlich die Kommunikation ist und wie vielfältig die vermittelte Sprache ist. Die Verwendung von besser geeigneten Medien führt zu höherer Effektivität der Aufgabenerfüllung [Daft\&Lengel 1986, S. 561]. Rice [1992] entwickelt die Media-Richness-Theorie für neue Medien fort. Reichwald et al. [1998a, S. 57] entwickeln daraus ein Media-RichnessModell für die Telekooperation (vgl. Abbildung 1). Face-to-Face Dialoge/Sitzungen haben den größten Medienreichtum; Briefpost und schriftliche Dokumentation den kleinsten. In Abhängigkeit davon, wie mehrdeutig die Telekooperationsaufgabe ist, sind andere Medien zu bevorzugen. Dabei ist es nicht so, daß reiche Medien per se 'besser' geeignet sind und arme

\footnotetext{
${ }^{1}$ Unsicherheit $=$ Uncertainty, Mehrdeutigkeit $=$ Equivocality.

2 Die Achse "Mehrdeutigkeit der Telekooperationsaufgabe" bezeichnen Reichwald et al. [1998a] als "Komplexität der Kommunikationsaufgabe". Im folgenden wird die ursprüngliche Bezeichnung von Daft und Lengel [1986] bevorzugt. Die Media Richness Theorie spricht selbst von 'Informationsaustausch', andere Autoren (z.B. [Reichwald et. 1998] und [Dennis\&Valacich 1998]) nur von 'Kommunikation'. Die Begriffe werden aber so umfassend gebraucht, daß zumindest die Kooperation noch mit darunter subsumiert werden kann.
} 
Medien schlechter. Vielmehr gibt es einen Bereich effektiver Kommunikation. Die Wahl zu reicher Medien führt zu einer Überkomplizierung ('Overcomplication') der Situation. Anstatt Fakten zu suchen, werden die Teilnehmer durch den Reichtum des Mediums abgelenkt; es wird interpretiert und möglicherweise Mehrdeutigkeit künstlich erzeugt. Die Verwendung zu armer Medien führt $\mathrm{zu}$ einer $\mathrm{zu}$ starken Vereinfachung ('Oversimplification'): Das Medium eignet sich nur für die Informationssuche, obwohl ein gemeinsames Verständnis durch gemeinsame Interpretation gefragt ist. Wegen mangelnden Feedbacks und Unpersönlichkeit des Mediums kann nicht gemeinsam interpretiert werden.

\section{Die TIP-Theorie}

Die TIP-Theorie (TIP für Time, Interaction, Performance) geht davon aus, daß real existierende Gruppen eine soziale und organisatorische Umgebung eingebunden sind und deshalb Gruppenverhalten nur aus einem Kontext heraus verständlich ist. Insbesondere ist Gruppenverhalten dynamisch und an einen zeitlichen Kontext gebunden. Von dieser Kontextabhängigkeit abstrahieren sehr viele Experimente und Theorien der Gruppenarbeit; deren Forschungsergebnisse sind deshalb auf echte Gruppen nur in sehr engen Grenzen anwendbar. Die TIP-Theorie besteht aus Aussagen zum Wesen von Gruppen, zur zeitlichen Strukturierung von Gruppenarbeit und zum Gruppeninteraktionsprozeß.

\section{a) Aussagen zum Wesen von Gruppen:}

Gruppen erfüllen simultan drei Funktionen:

1. Eine Produktionsfunktion, in der sie die ihnen übertragenen Aufgaben erfüllen und einen Beitrag zu ihrer Organisation leisten, indem sie ein Problem der Organisation lösen.

2. Eine Mitgliederunterstïtzungsfunktion, in der Beiträge an einzelne Gruppenmitglieder geleistet werden, z.B. indem einzelne Mitglieder eine persönliche Beziehung zueinander entwickeln.

3. Eine Gruppenwohlbefindensfunktion, in der Beiträge für die gesamte Gruppe geleistet werden, indem z.B. Mitglieder Rollen übernehmen und Verhaltensnormen entwickeln.

Bei ihren Aktivitäten nehmen die Gruppenmitglieder eine von vier Arbeitsmodi ein:

Modus I: Initialisierung, d.h. Zielfindung für das Projekt, für die Gruppe und für das einzelne Mitglied. Dies geschieht auf jeden Fall zu Beginn des Lebenszyklusses einer Gruppe, kann aber auch in späteren Phasen auftreten, z.B. wenn sich Aufgaben oder Gruppenmitgliedschaft ändert und deshalb die Ziele überdacht werden müssen.

Modus 2: Technische Problemlösung, d.h. das Lösen von alltäglichen organisatorischen und arbeitstechnischem Problemen im Projekt, wie das Projekt durchgeführt werden soll, wer welche Rolle hat und welche Personen welche Aufgaben übernehmen. Hierzu gehören auch Arbeitstechniken. Klassisch ist dies das Hauptinteresse der Gruppenforschung.

Modus 3: Konfliktlösung, d.h. wie können unterschiedliche Werte, Präferenzen, Entlohnung, Aufgaben und Interessen miteinander in Einklang gebracht werden.

Modus 4: Ausführung, d.h. die Aktivitäten, die erforderlich sind, um die gestellte Aufgabe zu erfüllen und die Ziele des Projekts, der Gruppe und der Individuen zu erreichen. Dies ist die "eigentliche Arbeit" der Gruppe. 
Die Modus und die Funktionen stehen orthogonal zueinander, d.h. jeder Modus findet in jeder Funktion seine Ausprägung. Dabei kann sich eine Gruppe zu einer Zeit für jede Funktion in einem anderen Modus befinden, d.h. z.B. für die Produktionsfunktion im Ausführungsmodus und für das Gruppenwohlbefinden in der Konfliktlösung.

Für die Mod gibt es keine feste zeitliche Reihenfolge der Aktivitäten, selbst wenn in der Regel zumindest Modus 1 und Modus 4 durchlaufen werden. Ziel der Gruppe ist es häufig, drekt von Modus $1 \mathrm{zu}$ Modus 4 überzugehen und dort $\mathrm{zu}$ verweilen; die Umstände erfordern es aber in der Praxis meist, daß immer wieder in die Modi 2 und 3 gewechselt werden muß. Eine Herausforderung für das externe Management, die Gruppenleitung und die Gruppenmitglieder ist es nun, gemeinsam einen möglichst effizienten Weg durch diese Modus zu finden. Dies sollte aber nicht über das Bestehen auf einem vorher geplanten Weg geschehen, sondern über das gezielte Hinterfragen, weshalb eine Gruppe das macht, was sie gerade macht.

\section{b) Aussagen zur zeitlichen Strukturierung von Gruppenarbeit}

Die Aussagen zur zeitlichen Strukturierung stellen ein Brücke von klassischer Gruppenforschung zu Erkenntnissen des Projektmanagements dar. Wesentliche relevante Merkmale sind dabei:

1. die zeitliche Abfolge der Arbeit; diese führt zu Problemen der Zeitplanung, Synchronisation und der Zuordnung von zeitlichen Ressourcen. Die Probleme erwachsen aus Unsicherheit über die Dauer und Terminierung von Ereignissen, aus den unterschiedlichen zeitlichen Interessen und Anforderungen der Akteure und aus der Knappheit von Zeit als Ressource. Deshalb gehört es zu den wesentlichen Gruppenaktivitäten, Deadlines zu vereinbaren und durchzusetzen, Normen für die reibungslose dynamische Zusammenarbeit $\mathrm{zu}$ etablieren und den Arbeitsfluß sowie die persönlichen Interaktionen zu regulieren, um ineffiziente oder ungleiche Anforderungen an die Ressourcen der Akteure aufzulösen.

2. Probleme bei der effizienten Zuordnung von Zeitperioden zu Aktivitätenbündeln. Subjektiv erfahrene Zeit ist nicht unbegrenzt teilbar und nicht jede Zeiteinheit ist homogen oder austauschbar. Nicht zu jeder Zeit kann jede Aktivität gemacht werden (auch wenn man von der inneren Logik der Aktivitäten absieht); z.B. sind manche Aktivitäten nachts nur sehr schwer durchführbar. Ebenso sind Aktivitäten nicht beliebig teilbar oder aggregierbar; es gibt vielmehr obere und untere Grenzen der sinnvollen Modularisierbarkeit. Dies macht die Zuordnung von Zeitperioden zu Aktivitätenbündeln zu einer besonderen Herausforderung für Gruppen.

3. Der Arbeitsrhythmus. Die Dauer und Intensität von mehreren Arbeitsprozessen müssen auf der Ebene eines Individuums, mehrerer Individuen sowie zwischen Gruppen und ihrer Außenwelt aufeinander abgestimmt werden. Der Arbeitsrhythmus ist wiederum stark abhängig vom Zeitdruck, unter dem Individuen oder Gruppen stehen. Er beeinflußt die Kommunikations- und Interaktionsmuster sowie Umfang und Qualität der Gruppenleistung.

\section{c) Aussagen zum Gruppeninteraktionsprozeß:}

Unter Gruppeninteraktionsprozeß wird der Arbeitsfluß auf der Microebene verstanden. Ein Interaktionsprozeß besteht aus Interaktionseinheiten. Eine Interaktionseinheit wird charakterisiert durch seinen Typ (z.B. Lösungsvorschlag, Ablehnung Lösungsvorschlag), seine Quelle und Adressaten sowie seine Zeit (relativer oder absoluter Beginn- und Endezeitpunkt). Ihre Bedeutung erhalten Interaktionseinheiten aus ihrem Kontext. Beispielsweise bedeutet ein 
neuer Lösungsvorschlag zum Ende des Problemlösungsprozesses (z.B. wenn eine Gruppe sich schon auf eine andere Lösung geeinigt hat) etwas anderes, als zu Beginn des Prozesses (wenn die Gruppe z.B. dankbar für jeden Vorschlag ist). Es ist auch wesentlich, ob die Interaktionseinheit zum derzeitigen Fokus der Gruppenarbeit paßt, oder nicht. Weiterhin hängt seine Bedeutung von dem Arbeitsmodus ab, in dem sich die Gruppe befindet. Mit diesen Aussagen wendet sich McGrath wiederum gegen $\mathrm{zu}$ simplistische Vorstellungen von Gruppenforschern, die - um einfacher zu Ergebnissen zu gelangen - Gruppenprozesse aus ihrem inneren Kontext lösen.

\section{Die Media-Synchronicity-Theorie}

Dennis und Valacich [1999] bescheinigen der Media-Richness-Theorie ein hohes Maß an Plausibilität, verweisen aber darauf, daß ihre empirischen Überprüfungen bisher nicht sehr überzeugend waren. Insbesondere basierten die Studien auf Wahrnehmungen der Eignung von Medien für Aufgaben, nicht aber auf echter Nutzung.

Dennis und Valacich [1999] argumentieren, daß der Grundansatz der Media-RichnessTheorie zu grob war: Die Media-Richness-Theorie geht davon aus, daß Charakteristika der Aufgabe und deren Anforderung an den Kontextreichtum eine optimale Medienwahl bestimmen. Es ist aber vielmehr die Art des Kooperationsprozesses und dessen Anforderung an die Informationsverarbeitungskapazität eines Mediums welche die Mediennutzung bestimmt. Ausgehend von den Kooperationsprozessen entwickeln sie eine eigene 'Media-SynchronicityTheorie'. Die Media-Synchronicity-Theorie unterscheidet zwei generische Kooperationsprozesse: Konvergente Prozesse und divergente Prozesse

In divergenten Prozessen werden Informationen verteilt; in konvergenten Prozessen werden sie verdichtet, um zu einem gemeinsamen Verständnis zu gelangen. Damit sind divergente Prozesse für die Reduktion von Unsicherheit geeignet, während konvergente Prozesse zur Reduktion von Mehrdeutigkeit beitragen. So ist hier die deutliche Verwandtschaft zur MediaRichness-Theorie in den Grundannahmen erkennbar.

Die Media-Synchronicity-Theorie erweitert aber die relevanten Medieneigenschaften: Nicht der 'Reichtum' eines Mediums ist entscheidend, sondern seine Synchronität: "Mediensynchronität ist das Ausmaß, in dem Individuen an der gleichen Aufgabe zur gleichen Zeit zusammenarbeiten, d.h. einen gemeinsamen Fokus haben " [Dennis\&Valacich 1999]. Das Potential von Medien macht die Media-Synchronicity-Theorie an fünf Faktoren fest:

Geschwindigkeit des Feedbacks: Wie schnell kann ein Kooperationspartner auf Nachrichten antworten?

Symbolvarietät: Auf wieviele Weisen kann Information übermittelt werden? Die Symbolvarietät übernimmt aus der Media Richness Theorie, wieviele Kanäle wieviele Hinweise welcher Art übermitteln können.

Parallelität: Auf wievielen Kanälen können wieviele Personen gleichzeitig kooperieren oder kommunizieren?

Überarbeitbarkeit: Wie umfassend und häufig kann der Sender seine Nachricht oder seinen Beitrag überarbeiten, bevor er ihn abschickt?

Wiederverwendbarkeit: Wie gut kann der Empfänger eine Nachricht oder einen Beitrag eines anderen wiederverwenden?

\footnotetext{
${ }^{3}$ Übersetzung durch den Autor.
} 
Abbildung 2 faßt die Medieneigenschaften und die Ansatzpunkte an einem Kommunikationskanal zusammen.

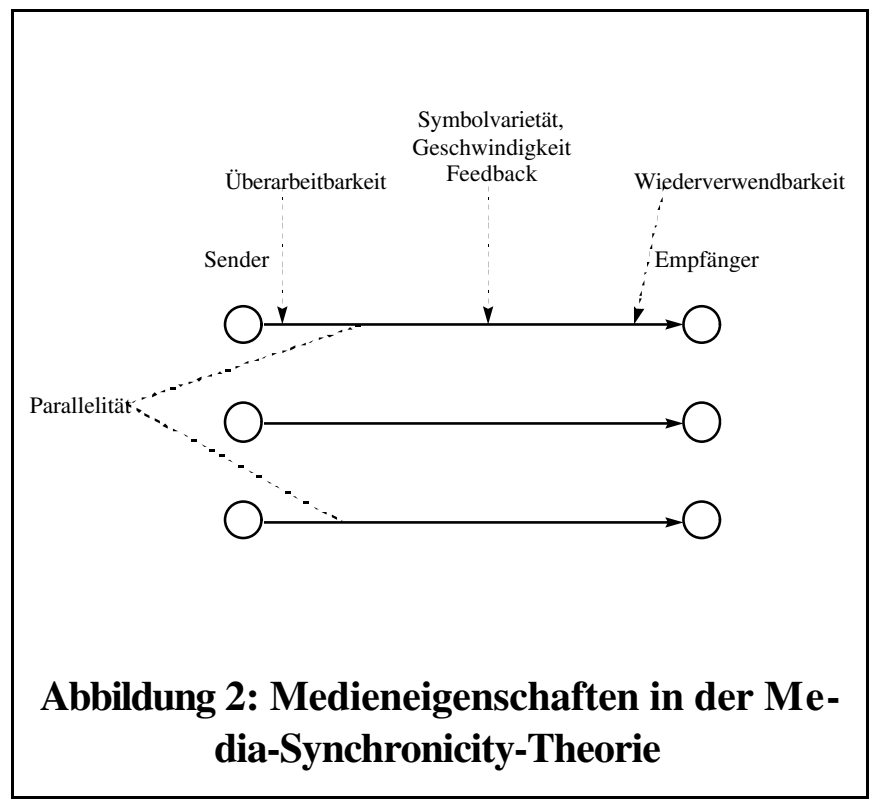

Die Überarbeitbarkeit wirkt auf den Sender, die Wiederverwendbarkeit auf den Empfänger. Parallelität beschreibt die Anzahl von Kommunikationskanälen und die Symbolvarietät, Geschwindigkeit und Feedback die Kapazität und Qualität des Kommunikationskanals.

Schon angesichts dieser fünf Faktoren wird unmittelbar deutlich, daß es absolut gesehen keine 'reichen' und 'armen' Medien geben kann: Überarbeitbarkeit und Feedback sind zwei gegenläufige Größen; Parallelität und Symbolvarietät haben keinen direkten inneren Zusammenhang. Im Kern der Betrachtung stehen die beiden Faktoren Feedback und Parallelität. Medien mit schnellem Feedback und geringer Parallelität ermöglichen hohe Synchronität; Medien mit langsamem Feedback und hoher Parallelität ermöglichen geringe Synchronität.

Zwischen den generischen Prozessen und den Medieneigenschaften bestehen folgende $\mathrm{Zu}$ sammenhänge: Für konvergente Prozesse sind Medien mit hoher Synchronität geeignet; für divergente Prozesse Medien mit geringer Synchronität. Generell führen Medien mit einer höheren Überarbeitbarkeit zu einer besseren Leistung; in konvergenten Prozessen führen Medien mit einer höheren Wiederverwendbarkeit zu einer besseren Leistung. Etablierte Gruppen benötigen weniger Synchronität als noch nicht etablierte Gruppen; im Laufe des Gruppenentwicklungsprozesses nimmt der Bedarf an Synchronität ab, da sich ein gemeinsames Grundverständnis entwickelt hat.

Neben die Prozeßtypen (divergent, konvergent) und die Kommunikationsverarbeitungskapazität eines Mediums tritt als dritte Dimension von Einflußfaktoren für die Medienwahl die drei Funktionen einer Gruppe (Produktion, Mitgliederunterstïtzung und Gruppenwohlbefinden). Es lassen sich in Abhängigkeit von den Funktionen und dem Alter der Gruppe (d.h. neuformiert oder schon länger zusammenarbeitend) unterschiedliche Empfehlungen zur Mediennutzung ableiten: Neue Gruppen benötigen mehr den Einsatz von Medien mit hoher Synchronität und hoher Symbolvielfalt, erfahrene Gruppen mehr den Einsatz von Medien mit geringer Synchronität. Insgesamt hängt der Erfolg einer länger andauernden Gruppenkooperation von dem richtigen Wechsel von Sychronitäts- und Mediennutzungsarrangements ab.

Die Media-Synchronicity-Theorie setzt dort an, wo die Media-Richness-Theorie ihre Schwächen hat: bei dem zu einfachen Bild der Gruppenarbeit. Die Media-Richness-Theorie unterscheidet nicht zwischen Zweipersonen-Kommunikation und Gruppenarbeit. Sie geht vielmehr bei der Betrachtung des Kommunikationsfalls implizit immer von zwei Personen aus (selbst wenn sie in ihrer Urform für mehr Personen formuliert ist!). Je größer die Gruppe wird, desto mehr wird dies zum Manko, weil typische Gruppenprobleme und das Potential der Technologie, diese zu lösen, nicht berücksichtigt werden. Die Media-Synchronicity-Theorie ergänzt deshalb die aus der Media-Richness-Theorie bekannten Faktoren 'Symbolvarietät' und 'Feedback' um die 'Parallelität'. Parallelität trägt zur Lösung einer Produktionsblockade bei, d.h. der begrenzten Redezeit, die ein einzelnes Gruppenmitglied in einer konventionellen Sitzung hat. 
Computerunterstützte Sitzungen ermöglichen Parallelität durch parallele Kommunikationskanäle und durch ein gemeinsames Material. Die Nutzung dieses Potentials setzt aber voraus, daß die Gruppe Sitzungen nicht nur zum Informationsaustausch durch Redebeiträge, sondern $\mathrm{zu}$ einem moderierten, workshopartigen gemeinsamen Arbeiten an einem gemeinsamen Material (vgl. [Schwabe 1995]) nutzt. Dann gewinnt auch die Überarbeitbarkeit und die Wiederverwendbarkeit von Informationen an Bedeutung. Damit ist aber schon eine Schwäche der Media-Synchronicity-Theorie benannt: Der Nutzen entspringt nicht einfach aus der Verwendung geeigneter Medien, sondern aus deren geschicktem Einsatz in einem moderierten Sitzungsprozeß oder - in Erweiterung dessen - in einem geschickt moderierten Einsatz von elektronischen Medien während der asynchronen Telekooperation. Dies setzt ein fundiertes Wissen über Gruppenprozesse und Moderationstechniken mindestens genauso voraus, wie das Wissen über Medieneigenschaften. Die Media-Synchronicity-Theorie hat somit bei der Berücksichtigung der Gruppeneigenschaften nur einen Schritt gemacht, wo mehrere nötig wären.

Dennoch gibt die Media-Synchronicity-Theorie schon in ihrer derzeitigen Fassung wertvolle Hinweise für die Gestaltung der Telekooperation in größeren Gruppen: Gruppen haben andere Probleme als Einzelpersonen; deshalb verliert der Medienreichtum seine einzigartige Stellung. Wichtiger wird es, Gruppen überhaupt produktiv arbeiten zu lassen und sie über die Zeit zu erhöhter Produktivität zu führen. Den Zusammenhang zwischen einem modifizierten Medienreichtum und Gruppenproduktivität aufzuweisen ist der große Verdienst der MediaSynchronicity-Theorie.

Aus beiden Theorien zur Medienwahl läßt sich schließen, daß bei computerunterstützter Gruppenarbeit auf geeignete Medien geachtet werden muß. Es besteht allerdings kein Konsens darüber, welche Faktoren die Medienwahl beeinflussen. Sind es Aufgabeneigenschaften (so die Media Richness Theorie) oder sind es generische Kooperationsprozesse (so die MediaSynchronicity-Theorie)? Das nachfolgende Unterkapitel versucht, auch darauf eine Antwort zu geben. Gemäß der 'Strukturationstheorie' kommt es nicht nur auf einzelne externe Faktoren an, welches Medium verwendet werden sollte, sondern es kommt darauf an, wie sich eine Gruppe Telekooperation aneignet. Damit wird die Medienwahl zu einem rückgekoppelten Prozeß und deutlich schwieriger $\mathrm{zu}$ beantworten, als dies die genannten Theorien zur Medienwahl suggerieren.

\section{Die Adaptive Structuration Theory}

DeSanctis\&Poole [1994] übertragen Ideen der Strukturationstheorie von Giddens [1984] 'Adaptive Structuration Theory' (AST) auf die Nutzung von Kooperationsmedien in Organisationen. Während bei Giddens das soziale System die Gesellschaft betrachtet, steht bei Poole und DeSanctis [1990] die Gruppe mit ihrer Technologie im Mittelpunkt der Betrachtung. Sie untersuchen, wie Technologie und Gruppenstrukturen Menschen in ihrem Handeln sowohl fördern als auch beschränken.

Nach der zentralen Hypothese der AST ist der Erfolg des Medieneinsatzes von komplexen, rückgekoppelten Wirkungsmechanismen abhängig: Bei gegebenem ${ }^{4}$ Kooperationsmedium und anderen Quellen für soziale Strukturen N1..Nk und einem idealen Aneignungsprozeß und Entscheidungsprozessen, die zur anliegenden Aufgabe passen, kommt es zu den erwünschten Ergebnissen der Nutzung von Kooperationsmedien [DeSanctis\&Poole 1994].

$\mathrm{Ob}$ eine Mediennutzung zu den gewünschten Ergebnissen führt, hängt mindestens davon ab, wie das Kooperationsmedium angeeignet wird und ob es zu der anliegenden Aufgabe paßt.

\footnotetext{
${ }^{4}$ Im Original fett.
} 
Was ein gewünschtes Ergebnis ist, kann z.B. über die Effizienz, Qualität oder den Konsens gemessen werden. Die Konstrukte der AST, ihre insgesamt 7 Hypothesen (H1 bis H7) und ihr Wirkungsgeflecht sind in Abbildung 3 zusammengefaßt.

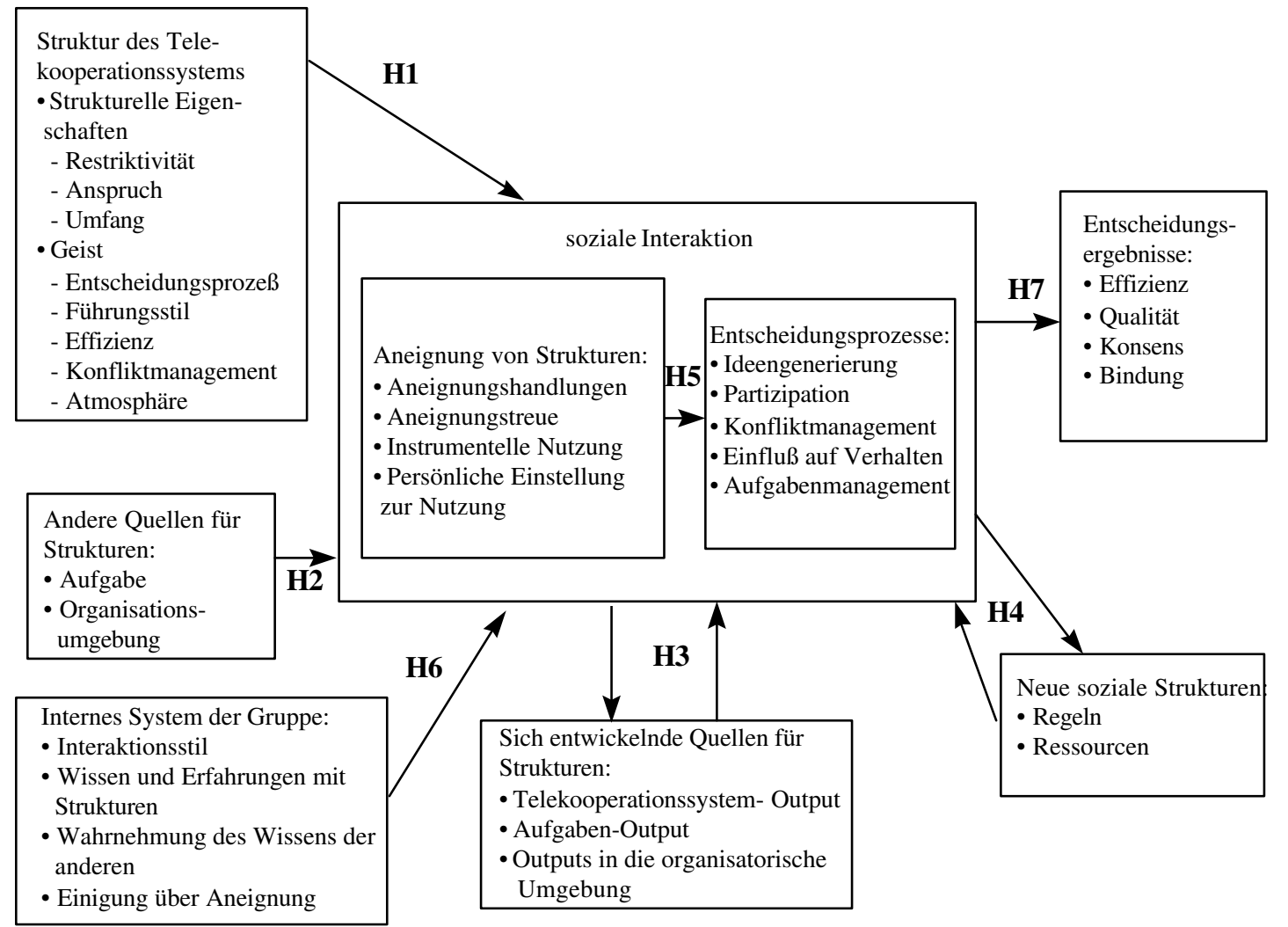

\section{Abbildung 3: Konstrukte und Hypothesen der Adaptive Structuration Theory nach DeSanctis\&Poole [1994, S. 132]}

Ein Kooperationsmedium wird durch seine Eigenschaften und seinen 'Geist' charakterisiert. In dem Maße, wie sich Kooperationsmedien in ihrem Geist und in ihren strukturellen Funktionen unterscheiden, werden unterschiedliche Formen der sozialen Interaktion durch die Technologie gefördert. 'Eigenschaften' sind die Funktionen, die das Kooperationsmedium vorsieht: dies kann die Möglichkeit sein, E-Mail zu versenden, gemeinsam Dokumente zu erstellen oder in einer computerunterstützten Sitzung Ideen zu sammeln. $\mathrm{Zu}$ den abstrakten Eigenschaften gehört, wie restriktiv das Kooperationsmedium ist, wie anspruchsvoll und ausgefeilt ('sophisticated') es ist, und welchen Funktionsumfang es abdeckt. Die Funktionen bringen Bedeutung ('Signifikation' im Sinne von Giddens) und Kontrolle ('Herrschaft' im Sinne von Giddens) in die Zusammenarbeit einer Gruppe.

Der 'Geist' eines Kooperationsmediums steht für die Absichten, Ziele und Werte, die hinter dem System stehen. Sie drücken sich aus in den geäußerten Erwartungen an den Umgang mit einem Kooperationsmedium und an der offiziellen Haltung dazu, wie die Funktionen eines Kooperationsmediums verwendet werden sollen: Wie sollte ein Entscheidungsprozeß mit Hilfe des Kooperationsmediums gestaltet werden? Welchen Führungsstil propagiert es? Welchen Stellenwert nimmt Effizienz ein? Welche Form des Konfliktmanagements ist vorgesehen? Und welche Atmosphäre der Zusammenarbeit wird unterstellt? Im Sinne von Giddens stellen sie die 'Legitimation' des Systems dar. Der Geist eines Systems kann auch zur Signifikation

\footnotetext{
5 Übersetzung durch den Autor; der Begriff 'Advanced Information Techonology' wurde durch Kooperationsmedium ersetzt.
} 
und Herrschaft beitragen. $\mathrm{Zu}$ dem Geist eines Kooperationsmediums tragen nicht nur die Absichten der Entwickler, sondern auch die der Personen, die das System einführen, und die der Nutzer bei. Je mehr Einigkeit bei allen Beteiligten über den Geist eines Systems besteht, desto größer ist sein Einfluß auf die Nutzung. Eigenschaften und Geist eines Systems bilden zusammen das strukturelle Potential eines Systems, mit denen Gruppen soziale Strukturen erzeugen können. In dem Maße, wie sich Telekooperationssysteme in ihrem Geist und in ihren strukturellen Funktionen unterscheiden, werden unterschiedliche Formen der sozialen Interaktion durch die Technologie gefördert (H1).

Ein Kooperationsmedium ist nur eine von vielen Quellen für Strukturen in Gruppen. Weitere Quellen für Strukturen sind die Aufgabe, die Organisation, die Informationen aus der Organisation, die Geschichte der Organisation, ihre Kultur etc. Die Nutzung eines Kooperationsmediums kann von allen abhängig sein $(\mathrm{H} 2)$. Neue Quellen für Strukturen entwickeln sich, indem die Technologie-, Aufgaben- und Umgebungsstrukturen während der sozialen Interaktion angewendet werden (H3). Auch durch die Nutzung eines Kooperationsmediums selbst entstehen neue Quellen von Strukturen. Wenn beispielsweise eine Gruppe einen internen elektronischen Markt für Aufgaben anlegt, ist dies eine Struktur, die aus der Nutzung heraus entsteht. Dieser elekronische Markt ist dann eine Quelle für soziale Strukturen. Somit entstehen neue soziale Strukturen bei der Interaktion in der Gruppe, indem die Regeln und Ressourcen eines Kooperationsmediums in einem gegebenen Kontext angeeignet und dann bei der Gruppeninteraktion über die Zeit reproduziert werden (H4).

Das Konzept der 'Aneignung' ist zentral für die AST: Bei der Aneignung wählt eine Gruppe bestimmte Funktionen eines Kooperationsmediums aus und paßt sie sich so an, wie sie es benötigt. Dabei können mit oder ohne Absicht auch die Eigenschaften des Systems verändert und der Geist des Systems getroffen oder von ihm abgewichen werden. Die Aneignung geschieht in einzelnen Aneignungsaktivitäten: Diese können schlüssiges Handeln oder Äußerungen zur Aneignung sein. Die Aneignung kann sich im Sinne des Designers von Kooperationsmedien, aber auch z.B. kreativ-erweiternd, ironisch-mißverstehend oder destruktivverneinend vollziehen. Mit einer Videokonferenzanlage läßt sich zum Beispiel auch der Zustand einer Kaffeemaschine übertragen, selbst wenn sie ursprünglich nicht dafür gedacht war.

Eine Aneignung ist kein einmaliger Akt, sondern ein laufender Prozeß. Die Gruppe füllt die sozialen Strukturen mit Leben, indem sie sie reproduziert. Die Strukturen ändern sich laufend fort. Eine Abnahme der Änderungsgeschwindigkeit ist möglich: Wenn ein Kooperationsmedium für eine Gruppe neu ist, dann sind viele unterschiedliche Aneignungsaktivitäten zu beobachten und die soziale Struktur kann noch stark variieren. Im Laufe der Zeit pendelt sich eine bestimmte Systemnutzung ein, und die sozialen Strukturen ändern sich nicht mehr so stark.

Gruppenentscheidungsprozesse hängen wiederum von der Aneignung des Kooperationsmediums ab (H5). Damit erklärt die AST auch die unterschiedlichen Ergebnisse der Forschung zu computerunterstïtzten Sitzungen bei scheinbar vergleichbaren Rahmenbedingungen (für eine Übersicht vgl. [Fjermestadt\&Hiltz 1999]). Die Art der Aneignung eines Kooperationsmediums hängt selbst vom internen System der Gruppe ab (H6). Das interne System einer Gruppe beinhaltet vier Größen:

1. Den Interaktionsstil der Gruppe: Eine Gruppe kann autoritär geführt werden oder z.B. ànen demokratischen Führungsstil haben; sie kann Konflikte offen austragen oder sie unter der Decke halten. 
2. Das Wissen und die Erfahrungen der Gruppe mit den Eigenschaften des Kooperationsmediums: Je nach ihrem Vorwissen und Können werden Gruppenmitglieder unterschiedliche Eigenschaften des Systems nutzen.

3. Die Bekanntheit der Strukturen: Je bekannter die Eigenschaften und der Geist des Systems sind, desto schneller kann die kritische Masse für eine Nutzung erreicht werden.

4. Die Einigkeit bezüglich der Nutzung: Je mehr Einigkeit bezüglich der Nutzung besteht, desto eher gelangt die Gruppe zu einem einheitlichen Nutzungsmuster und damit zur produktiven Arbeit.

Es ist schwierig, innerhalb der Strukturationstheorie zu kontingenten Hypothesen zu gelangen, die Aussagen über das Ergebnis der mit Telekooperation unterstützten Arbeit machen. Letztlich können weder das Kooperationsmedium noch die sozialen Strukturen außerhalb des Labors als gegeben angenommen werden, und ideale Aneignungsprozesse und passende Entscheidungsprozesse lassen sich auch schwer finden. Abbildung 3 suggeriert in den Hypothesen H2, H6 und H1 einseitige Ursache-Wirkung-Zusammenhänge, die so in der Realität ebenfalls nicht zu finden sind: Auch die organisatorischen Strukturen, das interne System der Gruppe und die Struktur des Kooperationsmediums mögen aus analytischen Gründen als konstant angenommen werden, sind es aber nicht immer, sondern können auch wiederum durch die Aneignung der Technologie verändert werden.

\section{Literatur}

Daft, R.; Lengel, R. (1984): Information Richness: A new approach to managerial behavior and organization design. In: Research in Organizational Behavior, Vol. 6 (1984), S. 191-233.

Daft, R.; Lengel, R. (1986): Organizational information requirements, media richness and structural design. In: Management Science, Vol 32 Nr. 5 1986, S. 554-571.

Dennis, A.; Valacich, J. (1999): Rethinking media richness: Towards a theory of media synchronicity. In: Proceedings of the $32^{\text {nd }}$ Annual Hawaii International Conference on System Sciences 1999, CD-ROM, IEEE Computer Society, Los Alamitos 10 pages.

DeSanctis, G.; Poole, M. (1994): Capturing the complexity in advanced technology use: Adaptive Structuration Theory. In: Organization Science, Vol. 5, No. 2 1994, S. 121 - 147.

Fjermestadt, J.; Hiltz, S. (1999): An Assessment of Group Support Systems Research. In: Proceedings of the $32^{\text {nd }}$ Annual Hawaii International Conference on System Sciences 1999, CD-ROM, IEEE Computer Society, Los Alamitos 10 pages.

Giddens, A. (1988): Die Konstitution der Gesellschaft - Grundzüge einer Theorie der Strukturierung, Campus, Frankfurt, New York 1988.

Lewe, H.(1995): CATeam und Produktivität. Gabler, Wiesbaden 1995.

McGrath, J. (1991): Time, interaction, and performance (TIP): A theory of groups. In: Small Group Research, Vol 22 Nr. 2 (1991), S.147-174.

Reichwald, R.; Möslein, K.; Sachenbacher, H.; Englberger, H.; Oldenburg, S. (1998a): Telekooperation - Verteilte Arbeits- und Organisationsformen, Springer, Heidelberg u.a. 1998a.

Schwabe, G. (1995): Objekte der Gruppenarbeit - ein Konzept für das Computer Aided Team. Gabler, Wiesbaden 1995. 